American Journal of Pharmaceutical Education 2017; 81 (7) Article 6181.

\title{
COMMENTARY
}

\section{Discrepancies in Lesbian, Gay, Bisexual, and Transgender Patient Care and How Pharmacists Can Support an Evolved Practice}

\author{
Erin Maxwell, Stephanie Salch, Monique Boliko, Genevieve Anakwe-Charles \\ University of North Carolina Eshelman School of Pharmacy, Chapel Hill, North Carolina \\ Submitted November 18, 2016; accepted June 14, 2017; published September 2017.
}

We live in an increasingly multicultural society with people from different ethnicities and beliefs. In recent years, we have witnessed a growing group of people who identify as having diverse sexual orientations and gender identities. Members of the lesbian, gay, bisexual, and transgender (LGBT) community have historically been marginalized by the health care industry. The disproportionate prevalence of LGBT health concerns and cultural prejudices may be overlooked by many practitioners. As pharmacists, we are in an optimal position to affect meaningful changes in how we treat, counsel, and interact with all our patients, including with those whose sexual orientation or gender identity differ from ours. It is important for student and practicing pharmacists alike to receive adequate education and training that identifies the role of a pharmacist in LGBT health and fosters culturally competent and equitable patient care. Clinical and cultural competence should be reflective of inclusive pharmacy programs that embrace and incorporate LGBT health. The objectives of this commentary are to identify the role of a pharmacist in LGBT health, recognize specific concerns with mental and sexual health, describe gender-transitioning pharmacotherapy, and discuss the current stance of LGBT health in pharmacy education.

Keywords: LGBT health, cultural competence, pharmacy curricula

\section{Pharmacy Encounters - A Personal Story}

A classmate recently shared a story with us about her first encounter with a transgender patient. She was working her shift at a large and busy community pharmacy when an individual dressed in feminine clothing approached the pickup counter. Assuming the customer to be a woman, she started her usual address of "Hello, ma'am" before realizing that the person was clearly, at least anatomically, a male.

She apologized for the mix-up and continued the transaction, eventually discovering that the patient was picking up a prescription for high-dose estradiol. Initially, she thought nothing of it - she considered herself to be open-minded and did not feel uncomfortable serving a transgender customer. The whole transaction looked to be commonplace until, as she was scanning the prescription, the patient asked her what factors should be considered when taking a medication at this high of a dose. Our classmate quickly realized she was in over her head - she had no idea how to counsel a patient who was transitioning with high-dose hormones. What should she tell the patient? Would the prescription interact with the

Corresponding Author: Erin Maxwell, UNC Eshelman School of Pharmacy, 301 Pharmacy Lane, Chapel Hill, NC 27599. Tel: 570-713-5388. E-mail: erin_maxwell@unc.edu medications the patient was on for other conditions? She didn't even know which pronoun to use when addressing the patient. He? She? The pharmacist-in-charge stepped in to help, but he also was unsure of his capabilities in this area. Even though the patient left with the prescription in hand, our classmate and her pharmacist felt as though they had provided, unintentionally, inadequate care.

\section{Addressing LGBT Health - The Pharmacist's Role}

While this is just one anecdote, our classmate's experience reflects a much larger and urgent issue: a provider's lack of confidence in their approach to LGBT health. During their first year, pharmacy students are taught the basics of patient counseling and empathetic service for all. As pharmacy school training progresses and students begin to learn more about drug pharmacology and therapeutics, they quickly learn appropriate indications, side effects, and other facts pertinent to typical patient education. Thus, it would be reasonable to infer that a third-year student, such as the one in our example, would feel confident in her ability to dispense a medication like estradiol. In this case, however, the patient was not "typical." This was a transgender patient who was using a drug therapy in a way that is not currently FDAapproved, and in a manner not typically addressed in school. 


\section{American Journal of Pharmaceutical Education 2017; 81 (7) Article 6181.}

As student pharmacists progress through their curriculum, they advance with the hope that they are being adequately trained to tackle the rigors of real-world patient care. However, they are probably not made aware that an estimated $28 \%$ of LGBT patients have reported postponing care due to the fear of discrimination. Similarly, $50 \%$ of LGBT patients have reported seeing providers who were unable to address their unique needs due to a lack of relevant knowledge. ${ }^{1}$ LGBT patients are not the only people who are noticing this gap in care. A 2016 survey delved into recent pharmacy graduates' understanding of LGBT patient care. Focusing on a group of community pharmacy residents in Maryland, the results of this survey found that while $98.2 \%$ of respondents felt they had a role to play in LGBT health care, $71.4 \%$ did not feel adequately educated about transgender issues, and $63.8 \%$ did not feel confident in their ability to provide appropriate treatment. ${ }^{2}$ Though this survey may not be reflective of all pharmacy practitioners, it raises salient questions about the adequacy of pharmacy curricula in addressing the needs of LGBT patients.

With a ubiquitous presence in nearly every American town, pharmacists are uniquely positioned to affect change in patient care. Widely considered to be one of the most trusted health care professions in the nation, pharmacists play a role that lends itself toward long-term relationships and well-earned trust. ${ }^{3}$ In fact, informal surveys have indicated that LGBT patients are more comfortable approaching their pharmacist as they do not fear the same repercussions they do with their primary provider. ${ }^{4}$ As a population at risk for certain health issues, such as sexually transmitted infections and mental illness, LGBT patients require our advocacy. ${ }^{5}$ It is important that pharmacists raise their awareness and expand their knowledge base about this cultural minority, and begin to break down the barriers to inclusive health care delivery.

\section{Addressing Health Concerns of LGBT Patients}

Mental Health. In the past half-century, America has seen extraordinary progress in the recognition of LGBT rights and advocacy. ${ }^{6}$ Synchronized advancement of mental health support, however, has not always been part of this evolution. Historically, the connection between homosexuality and psychopathology was largely based on religious, legal, and medical stigmas. Over time, various researchers, such as Freud and Hooker, redefined homosexuality as an intrinsic state of being; the medical community, including the American Psychiatric Association, recognized homosexuality as a treatable medical condition until the 1986 release of the DSM-III. ${ }^{7}$ Social psychologists have long pushed for the examination of the prevalence of mental disorders in LGBT patients, hoping that exposure will lead to meaningful change. One metaanalysis of randomized studies found that, over their lifetime, LGBT men and women were 2.5 times more likely to develop a mental disorder compared to straight, cisgender individuals. This same analysis hypothesizes that increased odds may result from "minority stress," a phenomenon caused by a hostile and stressful social environment created through systemic prejudice and discrimination. ${ }^{8}$ The true number of affected individuals is not well-established, but it is important nonetheless to note that serious mental health concerns continue to characterize this population.

As the prevalence of diagnosable psychiatric abnormalities, including depression, anxiety, and suicidal ideation, is so high among this population, it is important for pharmacists of all types to be aware of the ways they can provide support for LGBT patients. ${ }^{9}$ Additionally, a recent review of LGBT patients looked at the correlation between mental health and discrimination on the basis of sexual identity. ${ }^{10}$ Accessible health care professionals, such as pharmacists, have the capability to engage a large patient base. Important discussions addressing appropriate self-care practices, and perhaps the availability of local mental health resources, may play a pivotal role in getting at-risk patients in touch with opportunities for mental support. Preceptors might be encouraged to utilize their student pharmacists in these endeavors. As students are often trained in screening and addressing mental health concerns, they can help identify and assist with these undertakings. Being proactive about mental health advocacy can have a profound effect on minimizing mental health disparities, as long as we continue to recognize, and fill, the gaps.

Sexual Health. Pharmacists, especially those employed in the community setting, are very likely to encounter questions from LGBT patients regarding sexual health. It is important, then, that pharmacists are prepared to not only answer questions from their patients, but also be prescient with their own education so that they may identify opportunities to address the sexual health needs of this population.

LGBT patients are at higher risk for sexually transmitted infection and are more likely to forego preventive care. ${ }^{1}$ Whether this lapse of coverage stems from a lack of health insurance or a general fear of discrimination is unclear, but the correlation between inadequate prevention and prevalence of sexually transmitted infections (STIs) is well-defined. A recent publication from the Institute of Medicine (IOM) found that lesbian and bisexual women use preventive health services less frequently than their heterosexual counterparts. ${ }^{10}$ They are less likely, for 


\section{American Journal of Pharmaceutical Education 2017; 81 (7) Article 6181.}

example, to receive common health care services such as the human papilloma virus vaccine, cervical cancer screenings, and mammograms. ${ }^{9}$ LGBT men, too, are less likely to get recommended vaccinations. Additionally, they are less likely to make safe sexual health decisions. Men who have sex with men (MSM), while making up only an estimated $2 \%$ of the U.S. population, accounted for $61 \%$ of newly diagnosed HIV infections in 2009. Additional studies indicate that among MSM with HIV, there is a higher risk for hepatitis $\mathrm{B}$ and hepatitis $\mathrm{C}$ co-infections. ${ }^{11}$ Moving beyond preventive care, results from an IOM investigation indicate that nearly $40 \%$ of LGBT individuals report being sexually abused at some point during their adolescent or teen years. ${ }^{10}$ Providing an outlet for conversation and intervention for marginalized patients is essential to address these issues.

These statistics highlight a discouraging gap in LGBT health care, but pharmacists can provide a muchneeded service to help fulfill this need. Recognizing the vulnerability of this population allows pharmacists to be more thoughtful in how they care for these patients beyond medication dispensing and counseling. Pharmacists are trained to have conversations regarding birth control, preventive care, and the necessity of routine vaccinations; professionals should train themselves, and their co-workers, to ask these tough questions and have meaningful conversation with their LGBT patients. Students, too, should be encouraged to approach these conversations with an open mind and avoid making assumptions surrounding gender identity or sexual preference. Addressing these concerns with compassion will not only engender a trusting relationship and improve individual outcomes, but also allow pharmacists to make an impact on public health.

\section{Pharmacotherapy-induced Gender Transitions}

Transitioning, defined as a complex, individualized process resulting in changed gender identity, warrants a significant amount of support and guidance from providers. ${ }^{12}$ Pharmacists can fulfill these needs through counseling, monitoring, and managing expectations. As with all sensitive issues, pharmacists' care should include effective communication and familiarity with the appropriate terminology in order to cultivate a relationship based on trust and respect. In recognizing the unique barriers surrounding sexual transitions, the pharmacist must be equipped to provide culturally sensitive care while also delivering comprehensive counseling for any patient undergoing hormone reassignment therapy.

Hormone therapy can greatly enhance the transitioning patient's physical and mental well-being, but does come with its own risk. Testosterone and estrogens are the most frequently utilized therapies to achieve the goal of transitioning, but agents such anti-androgens and gonadotropin-releasing hormone $(\mathrm{GnRH})$ analogs may also be used as adjunct treatments. ${ }^{5}$ While these medications are generally considered safe and effective in "traditional" patients, exposure to the high doses required to achieve a successful transition are associated with increased risk of metabolic abnormalities and cardiovascular events. ${ }^{13}$ As medication experts, it is practical for pharmacists to refresh their understanding of hormone therapies and their potential uses in this kind of situation. Pharmacists should also make note of patients who may be undergoing a transition and work closely with other care providers to disseminate knowledge of appropriate dosing, monitoring, and suitable treatment goals to ensure a safer course of therapy. Receiving such care under the supervision of a pharmacist may help ensure a safer course of therapy. Additional roles for the pharmacist include adjudication of insurance claims and educating patients on the dangers associated with hormones purchased through illegal channels.

\section{LGBT Health in Pharmacy - Where Are We Now?}

Cultural competency education continues to be a focus in health care education across all disciplines. Recently, this type of training has begun to focus on the evolving needs of the LGBT community. Regardless of societal stigma or discrimination, it is imperative that health care providers be able to objectively address issues within this heavily diverse, and often overlooked, population. To fulfill the legacy of exceptional patient care, it is important that pharmacy schools continue to incorporate this type of competence training into their curricula.

Encouragingly, some professional schools, are already taking meaningful steps to encourage innovation in the care of LGBT patients. Schools hope to instill in their students a sense of responsibility and understanding of the LGBT population by incorporating relevant clinical skills and competency training into existing curricula. Current methods for integrating this type of training include LGBT patient panels, standardized patient scenarios, didactic seminars, and personal student reflections. ${ }^{14,15}$ Such endeavors to examine and increase student awareness of sensitive issues, such as addressing stigma and barriers to care, have been well received. A few pharmacy schools are also starting to shift their curricula to reflect a changing cultural environment. Northeastern University's pharmacy program recently assessed its pharmacy curriculum by examining the level of preparedness its third- and fourth-year student pharmacists exhibited for understanding LGBT care. Results from preliminary data suggested a low 


\section{American Journal of Pharmaceutical Education 2017; 81 (7) Article 6181.}

level of confidence and capability from these students; as a result, their program is currently working on implementing new didactic and experiential teaching methods to provide in-depth education on topics related to this patient population. ${ }^{16}$

Additionally, a variety of professional organizations have already established guidelines to serve as resources for practicing professionals. A 2014 Field Guide published by the Joint Commission (https://www.jointcommission. org/lgbt/) assembled a series of health-system strategies, practice examples, and resources for patient-centered LGBT community. There are also pharmacy-specific reference materials available for practicing pharmacists who seek continued education in this area. One such publication from the Human Rights Campaign (www.hrc.org) provides recommendations for establishing an inclusive environment within a pharmacy setting. The LGBT Health Education Center (www.lgbthealtheducation.org) at the Fenway Institute, in conjunction with the Institute of Medicine (IOM), provides comprehensive online resources for providers and patients alike. We encourage providers to investigate these resources and assess the environmental health of their own practice. The unique needs of LGBT patients underscore the necessity of the culturally competent pharmacist who is able to provide inclusive patientcentered care.

\section{CONCLUSION}

Providing optimal care for LGBT patients goes beyond clinical competence and encompasses an awareness and appreciation for cultural differences that characterize the LGBT community. We hope that student pharmacists will be able to start building this foundation for lifelong competency through the structure of their curricula. To do this, we must understand the antecedents of heterosexism, discrimination, and stigma this population must navigate on a daily basis and make a conscious effort to be engaged in the development of culturally sensitive education. Broadening perceptions and nurturing a well-rounded education through formal and informal instruction can help to cultivate a thoughtful, and inclusive, new generation of student pharmacists. We can, we should, and we will do more for this dynamic population.

\section{REFERENCES}

1. National Center for Transgender Equality, National Gay and Lesbian Task Force. Injustice at Every Turn: A Report of the National Transgender Discrimination Survey. http://www.thetaskforce.org/ static_html/downloads/reports/reports/ntds_full.pdf. Accessed September 15, 2016.

2. Leach C, Layson-Wolf C. Survey of community pharmacy residents' perceptions of transgender health management. $J \mathrm{Am}$ Pharm Assoc. 2016;56(4):441-445.

3. Gallup Poll: Honesty and Ethics of Professions. 2015. APhA. http://www.pharmacist.com/node/968170?is_sso_called=1.

Accessed Oct 2016.

4. Bonner L. Pharmacists can be accessible, trusted providers for transgender patients. Pharmacy Today. 2016;22(3):57.

5. Bishop BM. Pharmacotherapy considerations in the management of transgender patients: a brief review. Pharmacotherapy. 2015; 35(12):1130-1139.

6. Pandya A. Mental health as advocacy priority in the lesbian, gay, and transgender communities. J Psych Pract. 2014;20(3):225-227.

7. Department of Psychology, University of California Davis. Facts about Homosexuality and Mental Health. http://psychology.ucdavis.edu/ rainbow/html/facts_mental_health.html. Accessed September 25, 2016. 8. Meyer IH. Prejudice, social stress, \& mental health in lesbian, gay, and bisexual populations: conceptual issues and research evidence. Psychol Bull. 2003;129(5):674-697.

9. McNamara MC, Ng H. Best practices in LGBT care: a guide for primary care physicians. Cleve Clin J Med. 2016;83(7):531-541. 10. Institute of Medicine Committee on LGBT Health Issues and Research Gaps and Opportunities. The Health of Lesbian, Gay, Bisexual, and Transgender People: Building a Foundation for Better Understanding. Washington, DC: National Academies Press; 2011. 11. Lim FA, Brown DV, Justin Kim SM. Addressing health care disparities in the lesbian, gay, bisexual, and transgender population: a review of best practices. Am J Nurs. 2014;114(6):24-34.

12. Hyderi A, Angel J, Madison M, Perry LA, Hagshenas L. Transgender patients: providing sensitive care. J Fam Pract. July 2016;65(7):450-461.

13. Fernandez JD, Tannock LR. Metabolic effects of hormone therapy in transgender patients. Endocr Pract. 2016;22(4):383-388. 14. Brennan A, Barnsteiner J, Siantz ML, Cotter VT, Everett J. Lesbian, gay, bisexual, transgendered, or intersexed content for nursing curricula. J Prof Nurs. 2012;28(2):96-104.

15. Kelley L, Chou CL, Dibble SL, Robertson PA. A critical intervention in lesbian, gay, bisexual, and transgender health: Knowledge and attitude outcomes among second-year medical students. Teaching and Learning in Medicine. 2009; 20(3), 248-253. 16. Douglass MA, DeLeonardo K, Long A. Assessment of pharmacy student didactic and clinical preparedness surrounding LGBT and mental illness patient populations. Poster presented at American College of Clinical Pharmacy (ACCP) Annual Meeting; October 2016. Hollywood, FL. 